BMJ Nutrition,

Prevention \& Health

\section{Pasta meal intake in relation to risks of type 2 diabetes and atherosclerotic cardiovascular disease in postmenopausal women : findings from the Women's Health Initiative}

To cite: Huang M, Lo K, Li J, et al. Pasta meal intake in relation to risks of type 2 diabetes and atherosclerotic cardiovascular disease in postmenopausal women : findings from the Women's Health Initiative. BMJ

Nutrition, Prevention \& Health 2021;4:e000198. doi:10.1136/ bmjnph-2020-000198

- Additional supplemental material is published online only. To view, please visit the journal online (http://dx.doi.org/10. 1136/bmjnph-2020-000198).

For numbered affiliations see end of article.

Correspondence to

Professor Simin Liu, Department of Epidemiology, Brown University, 121 South Main St, Providence, RI 02903, USA; simin_liu@brown.edu

$\mathrm{MH}$ and $\mathrm{KL}$ contributed equally.

Received 31 October 2020

Revised 11 April 2021

Accepted 12 April 2021

Published Online First

30 April 2021

Check for updates

(c) Author(s) (or their employer(s)) 2021. Re-use permitted under CC BY-NC. No commercial re-use. See rights and permissions. Published by BMJ.

\section{ABSTRACT}

Objective To evaluate the association between pasta meal intake and long-term risk of developing diabetes or atherosclerotic cardiovascular disease (ASCVD, including coronary heart disease (CHD) and stroke) in postmenopausal women.

Design Prospective cohort study.

Setting Women's Health Initiative (WHI) in the USA. Participants 84555 postmenopausal women aged 50-79 in 1994, who were free of diabetes, ASCVD and cancer at baseline who were not in the dietary modification trial of the WHI, completed a validated food frequency questionnaire, and were evaluated for incident diabetes and ASCVD outcomes during the follow-up until 2010.

Main outcome measure Diabetes and ASCVD.

Results Cox proportional hazards models were used to estimate the association (HR) between quartiles of pasta meal consumption (residuals after adjusting for total energy) and the risk of incidence diabetes, CHD, stroke or ASCVD, accounting for potential confounding factors, with testing for linear trend. We then statistically evaluated the effect of substituting white bread or fried potato for pasta meal on disease risk. When comparing the highest to the lowest quartiles of residual pasta meal intake, we observed significantly reduced risk of ASCVD $(H R=0.89$, $95 \% \mathrm{Cl} 0.83$ to 0.96 , $\mathrm{p}$ trend $=0.002)$, stroke $(\mathrm{HR}=0.84$, $95 \% \mathrm{Cl} 0.75$ to 0.93 , p trend $=0.001$ ), $\mathrm{CHD}$ (HR=0.91, $95 \% \mathrm{Cl} 0.83$ to $1.00, \mathrm{p}$ trend $=0.058$ ) and no significant alteration in diabetes risk $(\mathrm{HR}=1.02,95 \% \mathrm{Cl} 0.96$ to 1.07 , $p$ trend $=0.328$ ). Replacing white bread or fried potato with pasta meal was statistically associated with decreased risk of stroke and ASCVD.

Conclusions Pasta meal intake did not have adverse effects on long-term diabetes risk and may be associated with significant reduced risk of stroke and ASCVD. The potential benefit of substituting pasta meal for other commonly consumed starchy foods on cardiometabolic outcomes warrants further investigation in additional high-quality and large prospective studies of diverse populations.

\section{What this paper adds}

The present study has found that higher pasta meal intake may be significantly associated with reduced long-term risk of stroke and atherosclerotic cardiovascular disease in postmenopausal women.

- Substituting pasta for an equal amount of fried potato or white bread could potentially be associated with lower risk of stroke and atherosclerotic cardiovascular disease.

\section{INTRODUCTION}

Among major sources of dietary carbohydrates, pasta has long been a staple food in diverse human cultures around the world. Interest in the health effects of pasta on the human body has steadily increased since the 1980s during a series of clinical studies of patients with diabetes showing that blood glucose response was remarkably reduced after ingesting spaghetti compared with white bread, ${ }^{1-4}$ potato $^{2-5}$ or rice. ${ }^{5}$ Many characteristics of pasta have been studied in relation to its glycaemic response. Notably, the structure (ie, viscosity, particle size and shape) of pasta appears to be more important in determining its glycaemic response than the types of cereal used in its production. ${ }^{6-8}$ It has also been found that consumption of pasta meal produced a lower postprandial insulin response than consumption of white bread in healthy subjects. ${ }^{6}$ Given the same amount, pasta appears to have lower glycaemic index (GI) as well as glycaemic load (GL) compared with other major sources of carbohydrates. ${ }^{4}$

The International Carbohydrate Quality Consortium reached consensus in 2015 that there was convincing evidence that low GI/ GL diets reduce the risk of type 2 diabetes and coronary heart disease (CHD), and GI 
represents another characteristic of carbohydrate foods apart from fibre and whole grain content. ${ }^{9}$ Dietary GL and GI have been linked to numerous cardiometabolic conditions ${ }^{10-14}$ and risk factors. ${ }^{15-18}$ Low-GI foods have been consistently associated with better glucose control in patients with diabetes. ${ }^{19}$ Since pasta has been shown to produce lower glycaemic response, it is then natural to hypothesise that consumption of pasta meal may have beneficial effects on the long-term risk of diabetes and atherosclerotic cardiovascular diseases (ASCVD, including CHD and stroke), given the same total carbohydrate consumption. However, few long-term studies have prospectively and directly investigated long-term average intake of pasta and the risk of developing diabetes, CHD, stroke and ASCVD. In the current study, we also aim to evaluate whether substituting other types of carbohydratedense food with pasta was associated with altered risk. To our knowledge, no other large-scale, long-term prospective cohort studies have specifically evaluated these relationships.

\section{METHODS}

\section{Study population}

The Women's Health Initiative (WHI) recruited a total of 161808 postmenopausal women aged 50-79 years at 40 clinical centres across the USA between 1993 and 1998, including a cohort of 93676 women in a prospective observational study (OS) and 68132 women in one or more of the following three clinical trials $(\mathrm{CTs})$ : the hormone therapy (HT) trial, the calcium and vitamin $\mathrm{D}(\mathrm{CaD})$ trial and the dietary modification $(\mathrm{DM})$ trial. We analysed baseline data from participants of the OS, and the HT and CaD trials of the WHI, for whom valid information was obtained from a validated 122-item food frequency questionnaire (FFQ) ${ }^{20}$ Participants of the DM trial were excluded due to potential major alterations in dietary behaviour after baseline. Additional exclusion criteria included: implausible total energy intake $(<600$ or $>5000 \mathrm{kcal} /$ day); prevalent diseases including diabetes, cardiovascular disease (CVD) and cancer that may alter dietary behaviours; measurements not available for outcomes of interest (incident diabetes, CHD or stroke); measurements not available for important covariates such as race and body mass index (BMI); and being underweight $\left(B M I<18.5 \mathrm{~kg} / \mathrm{m}^{2}\right)$ which may reflect underlying medical conditions (figure 1).

\section{Measurement of outcomes}

Incident diabetes was assessed via questionnaires at enrolment and each annual visit. Participants were asked if 'a doctor prescribed for the first time any of the following pills or treatments: pills for diabetes or insulin shots for diabetes' since their last medical update. Those who responded 'yes' were considered having been diagnosed with diabetes. Since these were all postmenopausal women, newly diagnosed diabetes cases were most likely type 2 diabetes cases, which have been shown to have a high validity. ${ }^{21}{ }^{22}$ Women who self-reported diabetes at baseline were excluded from the current analysis.

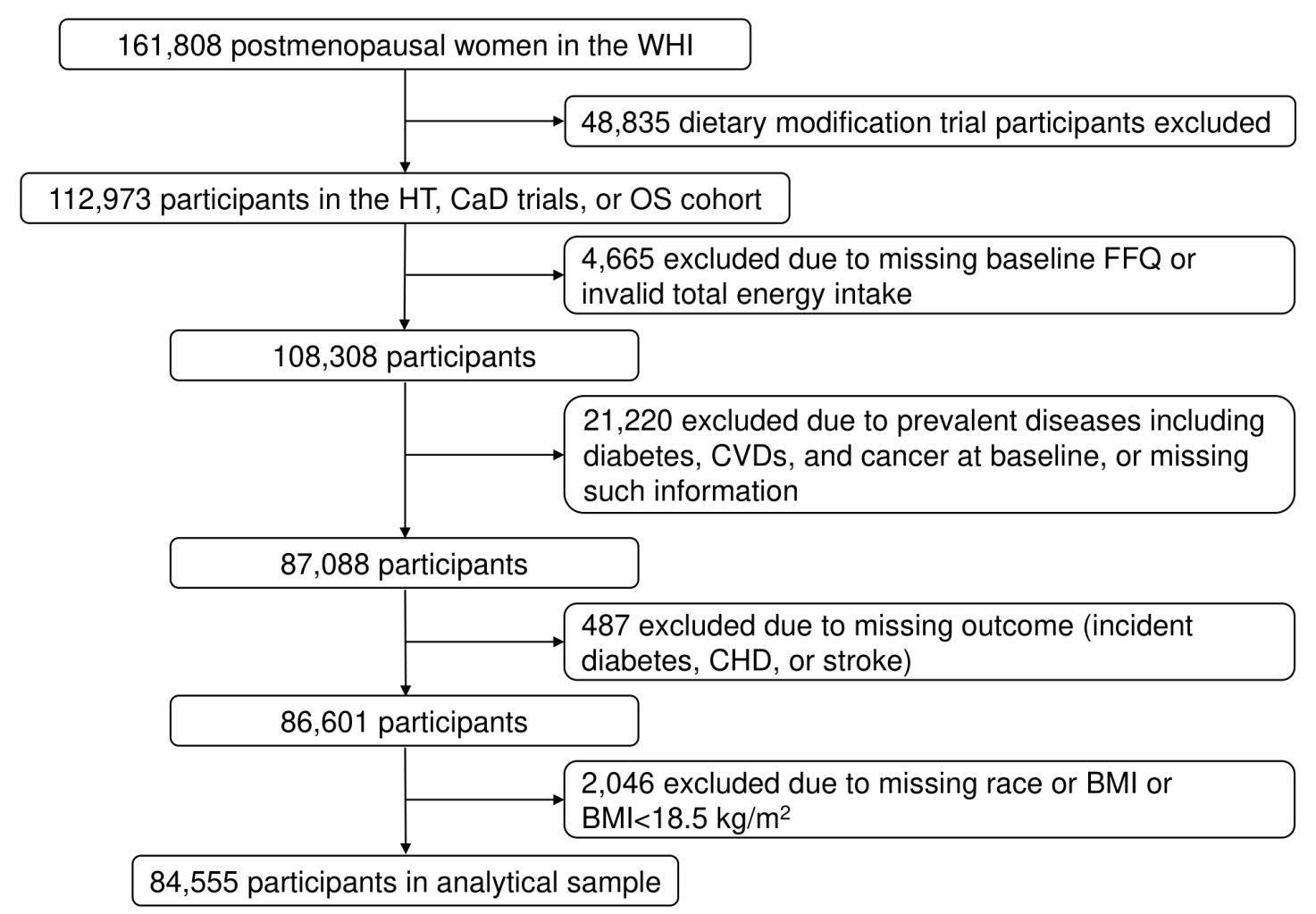

Figure 1 Analytical sample flow chart. BMI, body mass index; CaD, calcium and vitamin D; CHD, coronary heart disease; CVD, cardiovascular disease; FFQ, food frequency questionnaire; HT, hormone therapy; OS, observational study; WHI, Women's Health Initiative. 
Incident CHD was defined as the first occurrence of clinical myocardial infarction (MI), definite silent MI, or a death due to definite or possible CHD. Clinical MI and death were adjudicated for CT and OS participants during the core WHI study (until 2005) and Extension Study I (Ext1, until 2010).

Incident stroke was defined as the first occurrence of stroke or death due to cerebrovascular event. Stroke was adjudicated for CT and OS participants through Ext1. We further examined the concept of incident ASCVD as an outcome, which encompassed incident cases of CHD and stroke as defined above, according to the 2013 American College of Cardiology/American Heart Association Guideline on the Assessment of Cardiovascular Risk. ${ }^{23}$

\section{Measurement of pasta meal}

Using a validated semiquantitative $\mathrm{FFQ}^{20}{ }^{24}$ participants reported on the baseline FFQ how often they consumed each of the following forms of pasta during the past 3 months: macaroni and cheese/lasagna/noodles with a cream sauce, spaghetti or other noodles with meat sauce and spaghetti or other noodles with tomato sauce (and no meat), in frequency of medium servings (one cup). Nine frequency options were given, including: 'never or less than 1 per month', ' 1 per month', '2-3 per month', '1 per week', '2 per week', '3-4 per week', '5-6 per week', ' 1 per day' and ' $2+$ per day'. Participants also had the choices of 'small', 'medium' and 'large' for portion size per serving. The midpoint of the nine categories was used to compute the semicontinuous variables for these three forms of pasta meals, in number of medium servings per day. The sum of the three was used as a measure of total pasta meal intake. The residual method was then used where total pasta meal intake was first regressed on total energy intake, and the residuals added with mean total pasta meal intake were taken as a measure of pasta meal intake uncorrelated with total energy intake. ${ }^{25}$

In addition to residual total pasta meal intake, we also constructed two measures of pasta meal intake analogous to energy density standardisation: (1) the ratio of pasta to dietary GL was computed by dividing total pasta meal intake with total dietary GL and then multiplied by 100 ; (2) the ratio of pasta to total energy intake was computed by dividing total pasta meal intake with dietary total energy intake and then multiplied by 1000 . The respective scaling was done to obtain physiologically interpretable measurements. These two ratio measures were analysed in multivariable models in parallel with residual total pasta meal intake.

\section{Statistical analysis}

Baseline characteristics of participants included in the current analysis were described according to quartiles of residual total pasta meal intake. Means and SDs were generated as descriptive statistics for continuous variables, while frequencies and percentages were generated for categorical variables. Differences across pasta meal intake quartiles were tested by analysis of variance for continuous variables and by $\chi^{2}$ test for categorical variables.

Cox proportional hazards models were used to evaluate the association between residual total pasta meal intake and the risk of diabetes, CHD, stroke and ASCVD in terms of HRs and associated 95\% CIs, with study baseline as the origin of analysis and time to event or time to censoring as defined hereafter. For each disease condition of interest (diabetes/CHD/stroke/ASCVD), follow-up durations were calculated as the interval between baseline and the earliest of any of the following: (1) date of annual medical history update when new disease was reported, (2) date of last data collection from the main study if the participant did not enter the Extension Study, (3) date of last data collection from the Extension Study, or (4) date of reported death.

Residual pasta meal intake was analysed both in quartiles and as continuous variables. We also tested for linear trend after assigning the median of each quartile to the participants. The proportional hazards assumption was tested following standard procedures. ${ }^{26}$ We adjusted for the following potential confounding factors in model 1 : study group indicator (OS/HT/CaD), age (continuous), race/ethnicity (Caucasian, African-American, Hispanic, Asian/Pacific Islander or other) and region (Northeast, South, Midwest or West of the US). In model 2, we additionally adjusted for BMI (continuous, computed from weight and height measured by trained study staff), total energy intake and per cent energy intake from carbohydrates. In model 3 and the final model, we further adjusted for cigarette smoking (never, past or current), alcohol consumption (continuous), physical activity (in metabolic equivalent hours/week, continuous), and Healthy Eating Index (HEI 2005, ${ }^{27}$ continuous), and the respective family history of each outcome (diabetes, CHD, stroke or ASCVD). These potential confounding factors were chosen a priori based on current understanding of scientific literature and whether they could influence our exposure and outcomes of interest. Pasta to GL ratio and pasta to total energy ratio were analysed in similar procedures as the residual total pasta meal intake, in both continuous form and quartiles, and then tested for linear trend. We also evaluated the results adjusting for potential dietary confounders, including daily intake of fibre, total sugar, added sugar, non-whole grain, whole grain, frequency of eating a serving of vegetables and other major components in typical pasta meals (intake of total cheese and total tomato in medium servings per day).

As a sensitivity analysis, we included only pasta meals with spaghetti as the main carbohydrates source, as macaroni and cheese had been observed to have higher GI, ${ }^{7}$ analysed similarly to residual total pasta meal intake. In a second sensitivity analysis, we used age as the timescale in the Cox proportional hazards model instead of time to event. We also statistically tested for the substitutional effects of replacing pasta meal for the same amount of white bread or fried potato, measured with the same FFQ. 
To model such effects, if we take white bread as example, first the residual variable for white bread was created using the residual method; then residual total pasta meal intake and the sum of residual pasta and residual white bread were both included in model 3, so that the effect estimates for the residual pasta variable represented the estimated $\log (\mathrm{HR})$ for replacing one medium serving of white bread or fried potato by pasta, respectively, since the interpretation of the effect of the residual pasta variable was conditional on holding other covariates constant. ${ }^{28}$ All statistical analyses were conducted using R V.3.6.3. ${ }^{29}$

\section{RESULTS}

A total of 84555 participants of the WHI-OS, HT and CaD were included in the final analytical sample. Among these women, the median intake of pasta meal was 0.15 servings per day, or equivalently 1.04 servings per week, with the IQR from 0.08 to 0.26 servings per day (equivalently $0.54-$ 1.84 servings per week). This group of participants were on average 63.3 years old $(\mathrm{SD}=7.3)$, had an average BMI of $27.3 \mathrm{~kg} / \mathrm{m}^{2}(\mathrm{SD}=5.6)$, an average total energy intake of $1576.2 \mathrm{kcal} /$ day $(\mathrm{SD}=598.8)$ and an average total carbohydrates intake of $203.7 \mathrm{~g} /$ day $(\mathrm{SD}=78.0)$, which translated into an average of $52.4 \%$ energy from carbohydrates $(\mathrm{SD}=9.6)$. Eighty-five per cent of them were white and $6.8 \%$ were smokers at study baseline. Thirty per cent had a family history of diabetes, while $51.8 \%$ had a family history of CHD, $36.1 \%$ had a family of history of stroke and $65.2 \%$ had a family history of ASCVD.

Those in the higher quartiles of residual total pasta meal intake were on average younger, more likely to be white, less likely to be never smokers and more likely to have family history of diabetes and CHD, but not stroke or ASCVD. In terms of dietary intakes, women in the lowest and highest quartiles of residual pasta meal intake had on average higher total energy intake and GL, and higher intake of total carbohydrates, total sugar, added sugar intake, fibre and both whole and non-whole grains. Those in the lowest quartiles of residual total pasta meal intake had relatively higher alcohol intake, but lower per cent energy from carbohydrates and dietary quality as measured by HEI 2005. Physical activity levels and GI were relatively similar in magnitude across quartiles (table 1).

Results from the Cox proportional hazards models were summarised as follows by outcomes of interest. Residual total pasta meal intake across quartiles was not associated with risk of diabetes for postmenopausal women, after adjusting for age, race, region, study indicators, BMI, total energy intake, per cent energy from carbohydrates, smoking status, alcohol consumption, physical activity, HEI 2005 and family history of diabetes (model 3). Models 1-3 had similar results (table 2). Specifically, from model 3 (figure 2), compared with those in the lowest quartile of residual pasta meal intake, women in the second, third and highest intake quartiles had essentially no change in risk for incident diabetes (HR=0.97, 95\% CI 0.92 to 1.03 ; $\mathrm{HR}=1.00,95 \%$ CI 0.94 to 1.05 ; $\mathrm{HR}=1.02,95 \%$ CI 0.96 to 1.07 , respectively), and the linear trend was also not significant ( $p$ value for trend $=0.328$ ). Results were largely similar when examining quartiles of pasta to GL ratio or pasta to energy ratio.

For the CHD outcome, overall increased intake of pasta meal appeared to be associated with a decreased risk of developing CHD, especially when comparing women in the highest against the lowest quartile of residual total pasta meal intake (table 3). Specifically from model 3 (figure 2), compared with women in the lowest quartile of residual pasta meal intake, those in the second and third quartiles had no change in the risk of developing CHD $(\mathrm{HR}=0.99,95 \%$ CI 0.90 to 1.09 and $\mathrm{HR}=1.02,95 \%$ CI 0.93 to 1.12 , respectively), while women in the highest intake quartile had an estimated $9 \%$ reduction in risk $(\mathrm{HR}=0.91$, 95\% CI 0.83 to 1.00 ), while holding constant age, race, region, study indicators, BMI, total energy intake, per cent energy from carbohydrates, smoking status, alcohol consumption, physical activity, HEI 2005 and family history of CHD; with borderline significant linear trend ( $p$ value for trend $=0.058$ ). Results were largely similar across models 1-3 as well as when examining quartiles of pasta to GL ratio or pasta to energy ratio.

Women within the highest intake quartile of pasta meal had a significantly reduced risk of developing stroke compared with those in the lowest intake quartile of pasta ( $\mathrm{HR}=0.84,95 \%$ CI 0.75 to 0.93$)$, while those in the second and third quartiles had virtually no change in risk $(\mathrm{HR}=0.97,95 \%$ CI 0.88 to 1.08 and $\mathrm{HR}=1.00,95 \%$ CI 0.91 to 1.11, respectively) (table 4, figure 2). Testing for linear trend showed a significant inverse association ( $p$ value for trend $=0.001$ ), and results were highly consistent across models $1-3$ as well as when examining quartiles of pasta to GL ratio or pasta to energy ratio.

With ASCVD being a composite outcome of CHD and stroke, participants within the highest intake quartile of residual total pasta meal had an estimated $11 \%$ decreased risk of developing ASCVD ( $\mathrm{HR}=0.89,95 \%$ CI 0.83 to 0.96 ) compared with those in the lowest intake quartile in model 3, while those in the second and third quartiles had no change in risk ( $\mathrm{HR}=0.99,95 \%$ CI 0.92 to 1.06 and $\mathrm{HR}=1.03,95 \%$ CI 0.96 to 1.11 , respectively), with age, race, region, study indicators, BMI, total energy intake, per cent energy from carbohydrates, smoking status, alcohol consumption, physical activity, HEI 2005 and family history of ASCVD being constant (table 5, figure 2). Significant inverse trends were also observed, with $\mathrm{p}$ value for trend $=0.002$. Results were again highly consistent across models $1-3$ as well as when examining quartiles of pasta to GL ratio or pasta to energy ratio.

We also estimated the effects of one medium serving/ day increase in pasta meal intake variables on each disease of interest by entering the continuous variables of pasta meal intake into the models as exposure instead of quartiles. These analyses had similar results to the counterparts with quartiles as exposure, but the effect sizes seemed generally larger in magnitude (online supplemental table 1). Results were also robust to adjustment 
Table 1 Baseline characteristics of WHI participants in analytical sample by quartiles of energy-adjusted total pasta meal intake $(n=84555)$

\begin{tabular}{|c|c|c|c|c|c|}
\hline & \multicolumn{4}{|c|}{ Residual total pasta meal intake quartiles } & \multirow[b]{2}{*}{$P$ value } \\
\hline & Q1 & Q2 & Q3 & Q4 & \\
\hline Participants (n) & 21139 & 21139 & 21138 & 21139 & \\
\hline Mean (SD) (servings/day) & $0.03(0.07)$ & $0.13(0.07)$ & $0.21(0.08)$ & $0.45(0.27)$ & \\
\hline Median (servings/day) & 0.05 & 0.13 & 0.20 & 0.38 & \\
\hline (Q1, Q3) (servings/day) & $(0.01,0.08)$ & $(0.12,0.15)$ & $(0.18,0.23)$ & $(0.31,0.51)$ & \\
\hline \multicolumn{6}{|l|}{ OS or HT or CaD participant $(n)^{*}$} \\
\hline OS & 17175 & 17208 & 17237 & 17377 & \\
\hline HT & 3964 & 3931 & 3901 & 3762 & \\
\hline $\mathrm{CaD}$ & 2289 & 2257 & 2287 & 2204 & \\
\hline Age at baseline (years), mean (SD) & $64.0(7.3)$ & $63.9(7.3)$ & $63.2(7.2)$ & $61.9(7.2)$ & $<0.001$ \\
\hline BMI $\left(\mathrm{kg} / \mathrm{m}^{2}\right)$, mean (SD) & $27.4(5.7)$ & $27.0(5.5)$ & $27.1(5.5)$ & $27.6(5.8)$ & $<0.001$ \\
\hline Self-reported race/ethnicity, n (\%) & & & & & $<0.001$ \\
\hline White, non-Hispanic & $17396(82.3)$ & $17647(83.5)$ & $18257(86.4)$ & $18775(88.8)$ & \\
\hline African-American & $1660(7.9)$ & $1616(7.6)$ & $1402(6.6)$ & $1120(5.3)$ & \\
\hline Hispanic/Latino & $1042(4.9)$ & $842(4.0)$ & $700(3.3)$ & $626(3.0)$ & \\
\hline Asian/Pacific Islander & $710(3.4)$ & $749(3.5)$ & $501(2.4)$ & $295(1.4)$ & \\
\hline Other & $331(1.6)$ & $285(1.3)$ & $278(1.3)$ & $323(1.5)$ & \\
\hline Cigarette smoking status, n (\%) & & & & & $<0.001$ \\
\hline Never smoker & $10958(52.4)$ & $10950(52.4)$ & $10700(51.2)$ & $10036(48.1)$ & \\
\hline Past smoker & $8512(40.7)$ & $8503(40.7)$ & $8825(42.2)$ & $9425(45.1)$ & \\
\hline Current smoker & $1445(6.9)$ & $1433(6.9)$ & $1372(6.6)$ & $1415(6.8)$ & \\
\hline Alcohol consumption (g/day), mean (SD) & $7.0(14.3)$ & $5.6(10.5)$ & $5.3(9.9)$ & $5.6(10.5)$ & $<0.001$ \\
\hline Physical activity (METs/week), mean (SD) & $13.9(14.8)$ & $13.6(14.1)$ & $13.5(13.8)$ & $13.9(14.7)$ & 0.013 \\
\hline Total energy intake (kcal/day), mean (SD) & $1864.1(615.6)$ & $1414.0(494.8)$ & $1383.3(511.1)$ & $1643.4(631.1)$ & $<0.001$ \\
\hline Total carb intake (g/day), mean (SD) & $236.1(79.9)$ & $184.0(66.1)$ & $179.6(68.1)$ & $215.0(82.6)$ & $<0.001$ \\
\hline Per cent energy from carb, mean (SD) & $51.4(10.1)$ & $52.7(9.8)$ & $52.5(9.4)$ & $53.0(9.2)$ & $<0.001$ \\
\hline Total sugar intake (g/day), mean (SD) & $121.6(51.4)$ & $92.6(38.6)$ & $87.9(38.7)$ & $98.9(45.4)$ & $<0.001$ \\
\hline Added sugar intake (g/day), mean (SD) & $58.5(40.4)$ & $42.0(26.7)$ & $40.4(25.3)$ & $48.8(31.5)$ & $<0.001$ \\
\hline Total fibre (g/day), mean (SD) & $18.5(7.2)$ & $15.1(6.3)$ & $14.8(6.3)$ & $17.3(7.3)$ & $<0.001$ \\
\hline Glycaemic load, mean (SD) & $122.9(44.5)$ & $95.3(35.8)$ & $93.2(36.5)$ & $112.0(44.5)$ & $<0.001$ \\
\hline Glycaemic index, mean (SD) & $51.8(4.2)$ & $51.6(4.0)$ & $51.8(3.7)$ & $52.0(3.4)$ & $<0.001$ \\
\hline Whole grains, ounce equivalent/day, mean (SD) & $1.5(1.2)$ & $1.1(0.9)$ & $1.1(0.9)$ & $1.2(1.0)$ & $<0.001$ \\
\hline Non-whole grain, ounce equivalent/day, mean (SD) & $4.1(2.4)$ & $3.1(1.8)$ & $3.2(1.7)$ & $4.2(2.1)$ & $<0.001$ \\
\hline HEI 2005, mean (SD) & $67.8(11.4)$ & $69.5(10.6)$ & $69.5(10.2)$ & $69.0(10.1)$ & $<0.001$ \\
\hline Family history of diabetes, yes, n (\%) & $6380(30.3)$ & $6185(29.4)$ & $6237(29.6)$ & $6522(31.0)$ & 0.007 \\
\hline Family history of CHD, yes, $n(\%)$ & $10160(50.9)$ & $10366(51.7)$ & $10442(51.8)$ & 10639 (52.9) & 0.001 \\
\hline Family history of stroke, yes, $\mathrm{n}(\%)$ & $7782(37.0)$ & $7644(36.4)$ & $7515(35.7)$ & $7445(35.4)$ & 0.001 \\
\hline Family history of ASCVD, yes, $\mathrm{n}(\%)$ & $13466(65.1)$ & $13533(65.3)$ & $13473(65.0)$ & $13596(65.5)$ & 0.125 \\
\hline Incident diabetes, yes, n (\%) & $2689(12.7)$ & $2524(11.9)$ & $2618(12.4)$ & $2761(13.1)$ & 0.004 \\
\hline Incident CHD, yes, $\mathrm{n}(\%)$ & $1063(5.0)$ & $1061(5.0)$ & $1047(5.0)$ & $861(4.1)$ & $<0.001$ \\
\hline Incident stroke, yes, n (\%) & $889(4.2)$ & $842(4.0)$ & $822(3.9)$ & $620(2.9)$ & $<0.001$ \\
\hline Incident ASCVD, yes, n (\%) & $1842(8.7)$ & $1790(8.5)$ & $1775(8.4)$ & $1417(6.7)$ & $<0.001$ \\
\hline
\end{tabular}

${ }^{*}$ The WHI-HT and CaD trials had overlapping participants, while those in the WHI-OS were not in these trials by design, so the numbers may not add up to the total in each quartile.

ASCVD, atherosclerotic cardiovascular disease; BMI, body mass index; CaD, calcium and vitamin D; CHD, coronary heart disease; HEl, Healthy Eating Index; HT, hormone therapy; METs, metabolic equivalents; OS, observational study; Q, quartile; ;WHI, Women's Health Initiative. 
Table 2 Estimates of relative risk and $95 \%$ Cls of diabetes according to quartiles of pasta meal intake

Energy-adjusted residual total pasta meal intake quartiles

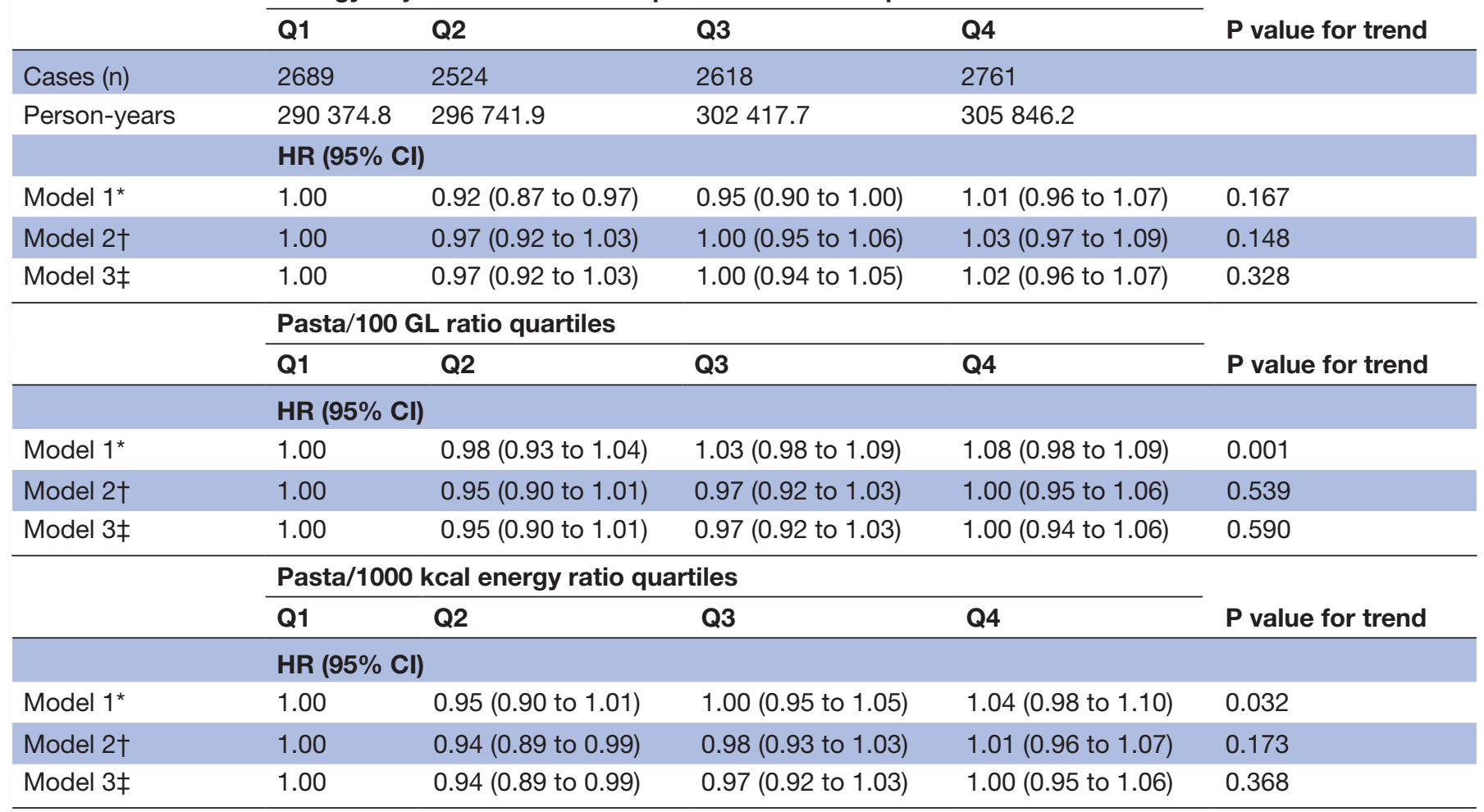

*Model 1 adjusted for age, race, region and study indicators.

†Model 2 adjusted for age, race, region, study indicators, body mass index (BMI), total energy intake and percent energy from carbohydrates. $\ddagger$ Model 3 adjusted for age, race, region, study indicators, BMI, total energy intake, per cent energy from carbohydrates, smoking status, alcohol consumption, physical activity, Healthy Eating Index (HEI) 2005 and family history of diabetes.

GL, glycaemic load.

for additional potential dietary confounders (online supplemental table 2).

When restricting to only spaghetti meals, the results were consistent with what we observed from the primary analyses (model 3) for each of the outcomes, in terms of effect estimates, CIs, as well as $\mathrm{p}$ value from trend analysis (online supplemental table 3). When using age as the timescale in the Cox proportional hazards model instead of time to event, there were also no substantial changes in model estimates compared with those regarding residual

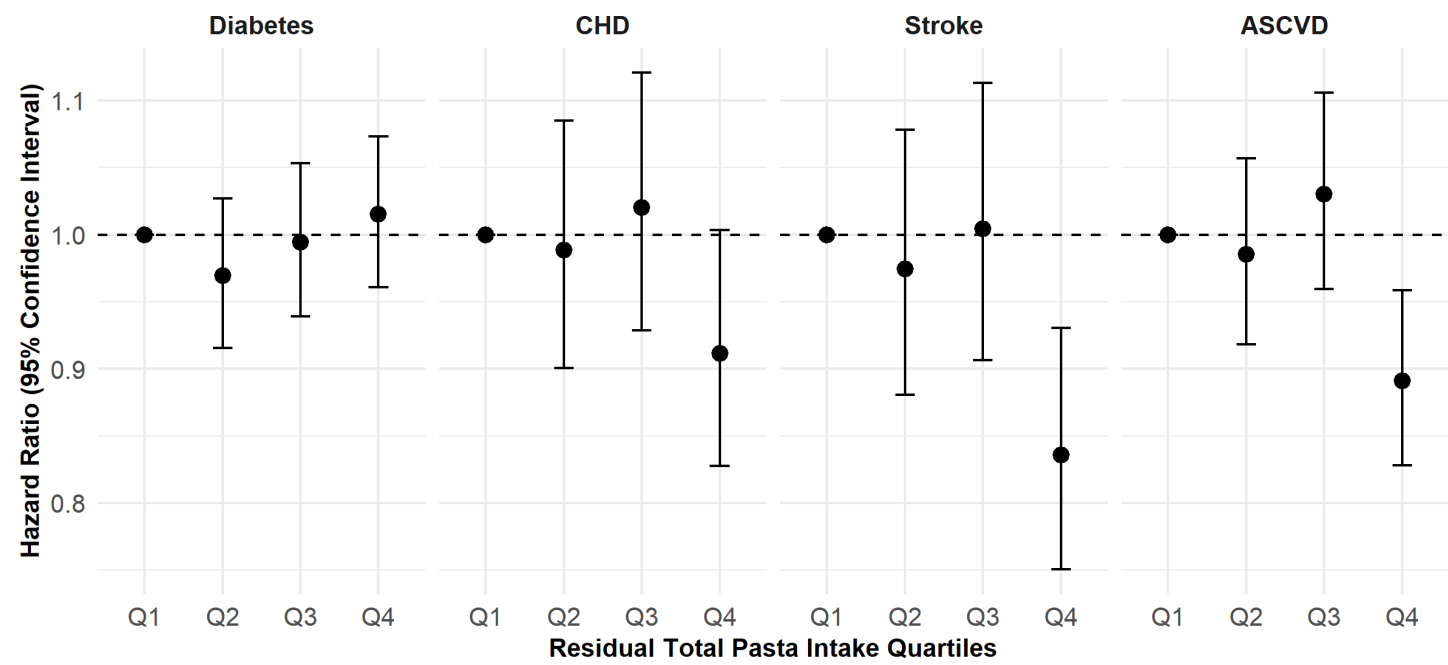

Figure 2 Estimates of relative risk and 95\% Cls of outcomes of interest according to quartiles of pasta meal intake from model 3 (adjusted for age, race, region, study indicators, body mass index (BMI), total energy intake, per cent energy from carbohydrates, smoking status, alcohol consumption, physical activity, Healthy Eating Index (HEI) 2005 and family history of the respective disease outcome). ASCVD, atherosclerotic cardiovascular disease; CHD, coronary heart disease. 
Table 3 Estimates of relative risk and 95\% Cls of CHD according to quartiles of pasta meal intake

\section{Energy-adjusted residual total pasta meal intake quartiles}

\begin{tabular}{|c|c|c|c|c|c|}
\hline & & \multirow[b]{2}{*}{$P$ value for trend } \\
\hline & Q1 & Q2 & Q3 & Q4 & \\
\hline Cases (n) & 1063 & 1061 & 1047 & 861 & \\
\hline Person-years & 303901.7 & 309179.7 & 315659.0 & 320927.2 & \\
\hline Model $1^{*}$ & 1.00 & 1.01 (0.93 to 1.10$)$ & 1.03 (0.95 to 1.12$)$ & 0.95 (0.86 to 1.03 ) & 0.207 \\
\hline Model 2† & 1.00 & 1.02 (0.93 to 1.11$)$ & 1.04 (0.95 to 1.14$)$ & 0.94 (0.86 to 1.03$)$ & 0.152 \\
\hline
\end{tabular}

\section{Pasta/100 GL ratio quartiles}

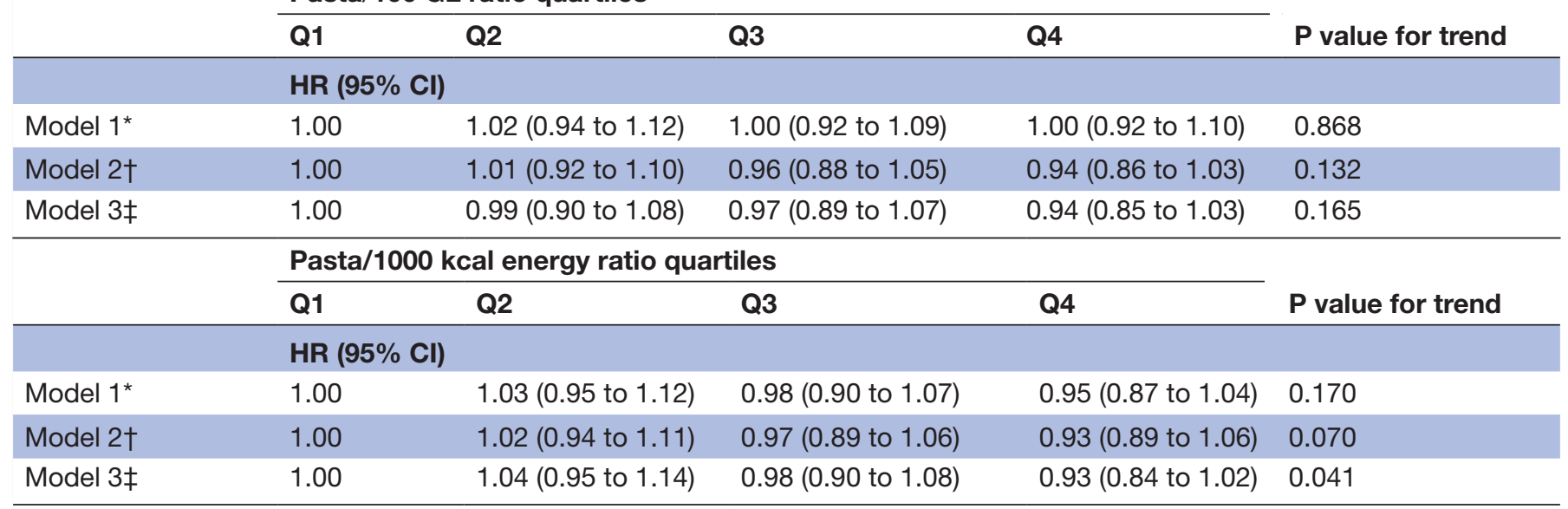

${ }^{*}$ Model 1 adjusted for age, race, region and study indicators.

†Model 2 adjusted for age, race, region, study indicators, body mass index (BMI), total energy intake and per cent energy from carbohydrates. ¥Model 3 adjusted for age, race, region, study indicators, BMI, total energy intake, per cent energy from carbohydrates, smoking status, alcohol consumption, physical activity, Healthy Eating Index (HEl) 2005 and family history of CHD.

CHD, coronary heart disease; GL, glycaemic load.

total pasta meal intake in tables 2-5 (online supplemental table 4). When statistically modelling the substitution effects, replacing fried potato with pasta meal was associated with a significant decreased risk of stroke $(\mathrm{HR}=0.57,95 \%$ CI 0.36 to 0.89$)$ and ASCVD ( $\mathrm{HR}=0.68$, $95 \%$ CI 0.50 to 0.93 ), as well as a reduction in the risk of diabetes $(\mathrm{HR}=0.78,95 \%$ CI 0.64 to 0.95$)$, while there was a suggestive reduction in the risk of CHD $(\mathrm{HR}=0.84$, $95 \%$ CI 0.55 to 1.28 ). Statistically substituting pasta meal for white bread was also associated with a significant reduction in the risk of stroke $(\mathrm{HR}=0.73,95 \%$ CI 0.59 to 0.92 ), and a borderline significant reduction in the risk of ASCVD (HR=0.88, 95\% CI 0.76 to 1.01 ), while the risk was unchanged for diabetes or CHD (table 6).

\section{DISCUSSION}

In this prospective analysis of 84555 postmenopausal women enrolled in the WHI followed until 2010, we observed a significant association between higher intakes of pasta meal and long-term risk of developing stroke and ASCVD, and a suggestive association between higher intakes of pasta meal and long-term risk of developing CHD, while no significant relation was observed between pasta meal intake and risk of developing diabetes. When we statistically estimated the substitutional effects of replacing other types of common starchy foods with pasta meal, we also found that substituting pasta for fried potato or white bread could potentially be associated with lower risk of stroke and ASCVD, and again a suggestive association was observed between substituting pasta meal for fried potato and lowered risk of CHD.

To our knowledge, our finding of the inverse relations between pasta meal intake and risk of stroke and ASCVD was the first time that such associations were reported. Measurements of body weight and adiposity are recognised as important risk factors for cardiometabolic diseases, and we considered our findings in the context of previous evidence relating pasta meal intake to these measurements. A cross-sectional analysis in two Italian cohorts, the Moli-sani study and the Italian Nutrition and Health Survey, which included over 20000 participants, demonstrated that higher pasta meal intake was associated with better adherence to Mediterranean diet, ${ }^{30}$ a dietary pattern with demonstrated cardiovascular benefit. ${ }^{31} 32$ The authors also found that higher pasta meal intake was associated with lower BMI, waist circumference, waist to hip ratio and lower prevalence of being overweight and obese, which was independent of adherence to Mediterranean diet and total energy intake. Similar crosssectional associations between higher pasta meal intake 
Table 4 Estimates of relative risk and $95 \%$ Cls of stroke according to quartiles of pasta meal intake

Energy-adjusted residual total pasta meal intake quartiles

\begin{tabular}{|c|c|c|c|c|c|}
\hline & Q1 & Q2 & Q3 & Q4 & $P$ value for trend \\
\hline Cases (n) & 889 & 842 & 822 & 620 & \\
\hline \multirow[t]{2}{*}{ Person-years } & 304807.1 & 310167.8 & 316120.4 & 322181.7 & \\
\hline & \multicolumn{4}{|c|}{ HR $(95 \%$ Cl) } & \\
\hline Model $1^{*}$ & 1.00 & 0.96 (0.87 to 1.06$)$ & 0.98 (0.89 to 1.08$)$ & 0.83 (0.75 to 0.92$)$ & 0.001 \\
\hline Model $2 \dagger$ & 1.00 & $0.98(0.89$ to 1.08$)$ & $1.00(0.90$ to 1.10$)$ & 0.84 (0.75 to 0.93$)$ & 0.001 \\
\hline \multirow[t]{4}{*}{ Model 3‡ } & 1.00 & 0.97 (0.88 to 1.08$)$ & $1.00(0.91$ to 1.11$)$ & 0.84 (0.75 to 0.93$)$ & 0.001 \\
\hline & \multicolumn{4}{|c|}{ Pasta/100 GL ratio quartiles } & \\
\hline & Q1 & Q2 & Q3 & Q4 & $P$ value for trend \\
\hline & HR $(95 \%$ C & & & & \\
\hline Model $1^{\star}$ & 1.00 & 0.98 (0.89 to 1.08$)$ & 0.99 (0.90 to 1.09$)$ & 0.87 (0.79 to 0.97$)$ & 0.009 \\
\hline Model $2 \dagger$ & 1.00 & 0.96 (0.88 to 1.06$)$ & $0.96(0.87$ to 1.06$)$ & 0.84 (0.76 to 0.93$)$ & 0.001 \\
\hline \multirow[t]{4}{*}{ Model 3ł } & 1.00 & 0.97 (0.88 to 1.07$)$ & 0.97 (0.88 to 1.08$)$ & 0.85 (0.77 to 0.95$)$ & 0.003 \\
\hline & \multicolumn{4}{|c|}{ Pasta/1000 kcal energy ratio quartiles } & \\
\hline & Q1 & Q2 & Q3 & Q4 & $P$ value for trend \\
\hline & \multicolumn{4}{|c|}{ HR (95\% Cl) } & \\
\hline Model $1^{*}$ & 1.00 & 0.91 (0.83 to 1.01$)$ & 1.00 (0.91 to 1.09$)$ & 0.84 (0.76 to 0.93$)$ & 0.003 \\
\hline Model $2 \dagger$ & 1.00 & 0.91 (0.82 to 1.00$)$ & 0.99 (0.90 to 1.09$)$ & 0.83 (0.75 to 0.92$)$ & 0.003 \\
\hline Model 3‡ & 1.00 & 0.91 (0.82 to 1.00$)$ & $1.00(0.91 .1 .10)$ & 0.84 (0.75 to 0.93$)$ & 0.005 \\
\hline
\end{tabular}

*Model 1 adjusted for age, race, region and study indicators.

†Model 2 adjusted for age, race, region, study indicators, body mass index (BMI), total energy intake and per cent energy from carbohydrates. $\ddagger$ Model 3 adjusted for age, race, region, study indicators, BMI, total energy intake, per cent energy from carbohydrates, smoking status, alcohol consumption, physical activity, Healthy Eating Index (HEI) 2005 and family history of stroke.

GL, glycaemic load.

and lower BMI were observed in US adults as part of the International Study of Macronutrients/Micronutrients and Blood Pressure study. ${ }^{33}$ From another analysis of the same study where 17 population samples in four countries (China, Japan, UK, USA) were included, it was also found that individuals with low risk of developing CVD, as classified by favourable profile of CVD risk factors, reported higher intake of pasta meal, among other food items such as fruits, vegetables and fish. Interestingly, in studies of empirically derived dietary patterns, pasta has sometimes been classified into unhealthful patterns, ${ }^{34} 35$ or represented on both healthful and unhealthful patterns, ${ }^{36}$ possibly due to correlations in intake level with certain groups of healthful or unhealthful food items. Therefore, the potential benefit of consuming pasta should be considered in the context of an overall healthy dietary pattern, for instance, the Mediterranean diet.

Dietary GI and GL have been positively linked with the risk of a number of cardiometabolic diseases, including metabolic syndrome, ${ }^{10}$ type 2 diabetes $,{ }^{11-133738} \mathrm{CHD},{ }^{1439-41}$ stroke ${ }^{4243}$ and risk factors including triglycerides, ${ }^{15}{ }^{16}$ highdensity lipoprotein cholesterol, ${ }^{15-17}$ low-density lipoprotein cholesterol ${ }^{16}$ and high-sensitivity C-reactive protein. ${ }^{18}$ In patients with diabetes, low-GI foods have been consistently associated with better glucose control in the blood. ${ }^{19}$ We consider the low-GI nature of pasta a major reason why we observed inverse associations with risk of stroke and ASCVD, given the assumption that pasta was the primary component of the pasta meals that we included in assessing the exposure, which remained unchanged when pasta meal intake was standardised by total energy intake or GL, and the benefit remained when statistically replacing white bread or fried potato, both with high GI, with pasta. Moreover, previous systematic reviews assessing the effect of pasta on body weight and biochemical intermediaries indicated that pasta meal intake did not adversely affect adiposity and reduced body weight and BMI, although a large amount of unexplained heterogeneities were found among previous studies. ${ }^{44}$ In the TOSCA.IT study, glucose control, BMI, low-density lipoprotein cholesterol, triglycerides and the prevalence of obesity were not significantly different across the quartiles of pasta meal intake in people with type 2 diabetes. ${ }^{45}$ Taken together, available evidence indicates that greater intake of pasta meal may have a beneficial effect on cardiometabolic risk profile particularly in affecting ASCVD risk. In the context of unchanged total carbohydrate intake and total energy intake, pasta meal intake appeared not to be associated with type 2 diabetes risk.

Ours was the first prospective study to report potential inverse associations between pasta meal intake and 
Table 5 Estimates of relative risk and 95\% Cls of ASCVD according to quartiles of pasta meal intake

Residual total pasta meal intake quartiles

\begin{tabular}{|c|c|c|c|c|c|}
\hline & Q1 & Q2 & Q3 & Q4 & $P$ value for trend \\
\hline Cases (n) & 1842 & 1790 & 1775 & 1417 & \\
\hline \multirow[t]{2}{*}{ Person-years } & 300015.7 & 305683.9 & 311598.6 & 318080.3 & \\
\hline & \multicolumn{5}{|c|}{ HR (95\% Cl) } \\
\hline Model $1^{*}$ & 1.00 & 0.98 (0.92 to 1.05$)$ & $1.02(0.95$ to 1.08$)$ & 0.90 (0.84 to 0.97$)$ & 0.005 \\
\hline Model $2 \dagger$ & 1.00 & $1.00(0.93$ to 1.07$)$ & $1.03(0.96$ to 1.10$)$ & 0.90 (0.84 to 0.97$)$ & 0.003 \\
\hline \multirow[t]{4}{*}{ Model 3ł } & 1.00 & 0.99 (0.92 to 1.06$)$ & 1.03 (0.96 to 1.11$)$ & 0.89 (0.83 to 0.96$)$ & 0.002 \\
\hline & \multicolumn{4}{|c|}{ Pasta/100 GL ratio quartiles } & \\
\hline & Q1 & Q2 & Q3 & Q4 & $P$ value for trend \\
\hline & \multicolumn{5}{|c|}{ HR $(95 \%$ Cl) } \\
\hline Model $1^{*}$ & 1.00 & 1.01 (0.94 to 1.08$)$ & $1.01(0.95$ to 1.08$)$ & 0.95 (0.89 to 1.02$)$ & 0.111 \\
\hline Model $2 \dagger$ & 1.00 & 0.99 (0.93 to 1.06$)$ & 0.98 (0.92 to 1.05$)$ & 0.90 (0.84 to 0.97$)$ & 0.003 \\
\hline \multirow[t]{4}{*}{ Model 3ł } & 1.00 & 1.00 (0.93 to 1.07$)$ & 0.99 (0.93 to 1.07$)$ & 0.91 (0.85 to 0.98$)$ & 0.008 \\
\hline & \multicolumn{4}{|c|}{ Pasta/1000 kcal energy ratio quartiles } & \\
\hline & Q1 & Q2 & Q3 & Q4 & $P$ value for trend \\
\hline & \multicolumn{4}{|c|}{ HR (95\% Cl) } & \\
\hline Model 1* & 1.00 & $0.98(0.92$ to 1.04$)$ & $1.00(0.94$ to 1.07$)$ & 0.91 (0.85 to 0.98$)$ & 0.010 \\
\hline Model $2 \dagger$ & 1.00 & 0.97 (0.91 to 1.03$)$ & 0.99 (0.93 to 1.06$)$ & 0.90 (0.84 to 0.96$)$ & 0.004 \\
\hline Model $3 \ddagger$ & 1.00 & $0.98(0.92$ to 1.05$)$ & 1.01 (0.94 to 1.08$)$ & 0.90 (0.84 to 0.97$)$ & 0.004 \\
\hline
\end{tabular}

*Model 1 adjusted for age, race, region and study indicators.

†Model 2 adjusted for age, race, region, study indicators, body mass index (BMI), total energy intake and per cent energy from carbohydrates. ¥Model 3 adjusted for age, race, region, study indicators, BMI, total energy intake, per cent energy from carbohydrates, smoking status, alcohol consumption, physical activity, Healthy Eating Index (HEI) 2005 and family history of coronary heart disease (CHD) and stroke.

ASCVD, atherosclerotic cardiovascular disease; GL, glycaemic load.

long-term incident cardiometabolic disease risk in a wellcharacterised national cohort of postmenopausal women with high-quality follow-up. However, several limitations should be considered when interpreting the results. Total intake of pasta meal in this study was measured by summing the semiquantitative intake frequencies of pasta

Table 6 Estimates of relative risk and 95\% Cls for specific diseases of interest by statistically substituting one medium serving of pasta meal for other starch-dense foods from model $3^{*}$

\begin{tabular}{lll}
\hline & $\begin{array}{l}\text { Pasta replacing fried } \\
\text { potato } \\
\text { HR }(95 \% \mathrm{Cl})\end{array}$ & $\begin{array}{l}\text { Pasta replacing } \\
\text { white bread } \\
\text { HR }(95 \% \mathrm{Cl})\end{array}$ \\
\hline Diabetes & $0.78(0.64$ to 0.95$)$ & $1.03(0.93$ to 1.14$)$ \\
CHD & $0.84(0.55$ to 1.28$)$ & $0.97(0.80$ to 1.17$)$ \\
Stroke & $0.57(0.36$ to 0.89$)$ & $0.73(0.59$ to 0.92$)$ \\
\hline ASCVD & $0.68(0.50$ to 0.93$)$ & $0.88(0.76$ to 1.01$)$
\end{tabular}

*Model adjusted for age, race, region, study indicators, body mass index (BMI), total energy intake, per cent energy from carbohydrates, smoking status, alcohol consumption, physical activity, Healthy Eating Index (HEI) 2005 and family history of the respective outcome.

ASCVD, atherosclerotic cardiovascular disease; CHD, coronary heart disease. meals which had other commonly used ingredients such as cheese or tomato. Since the data were collected via FFQ we could not obtain information on the proportion of pasta content within a specific pasta meal, and how much the additional ingredients of the meal may influence its association with cardiometabolic outcomes. To the extent possible, we accounted for other aspects of participants' diet by adjusting for their overall quality of dietary intake. Additional adjustment for total intake of cheese or tomato also did not substantially change the results. The source of production or the types of pasta consumed (eg, regular or whole grain or legume based, or the type of flour used in manufacturing) may lead to different GI or GL. We attempted a sensitivity analysis excluding types of pasta meal with potentially different GL such as macaroni and cheese, and the results were similar to those in the primary analysis. Finally, the findings presented here stemmed from a cohort of postmenopausal women and the overall consumption of pasta meal was relatively low (average of 1 serving/week), which may have partially influenced the observed lack of association with diabetes risk, and further analysis within cohorts of men or both sex with higher levels of consumption should be conducted to evaluate the prospective associations between pasta meal intake and risk of cardiometabolic 
disease. Further work to confirm these observations is warranted in additional high-quality prospective cohort studies of diverse populations.

In conclusion, in this large and prospective cohort of postmenopausal women followed for up to 20 years, pasta meal intake did not have any adverse effects on risk of diabetes and may be associated with significant reduced risk of stroke and ASCVD. Substituting pasta meal for other commonly consumed starchy foods such as fried potato or white bread may possibly represent a feasible and easy-to-implement method of DM to improve cardiometabolic outcomes.

\section{Author affiliations}

${ }^{1}$ Department of Epidemiology, Brown University School of Public Health, Providence, Rhode Island, USA

${ }^{2}$ Center for Global Cardiometabolic Health, Brown University, Providence, Rhode Island, USA

${ }^{3}$ Channing Division of Network Medicine, Brigham and Women's Hospital and Harvard Medical School, Boston, Massachusetts, USA

${ }^{4}$ Department of Applied Biology and Chemical Technology, The Hong Kong

Polytechnic University, Hung Hom, Hong Kong, China

${ }^{5}$ Center for International Health, Guangdong Provincial People's Hospital, Guangdong Academy of Medical Sciences, Guangzhou, Guangdong, China ${ }^{6}$ Department of Family Medicine and Public Health, San Diego School of Medicine, University of California San Diego, La Jolla, California, USA

${ }^{7}$ Center for Long term Services and Support, Providence VA Medical Center, Alpert Medical School, Brown University School of Public Health and the Miriam Hospital Center for Cardiac Fitness, Providence, Rhode Island, USA

${ }^{8}$ Division of Endocrinology, Department of Medicine, and Division of Cardiothoracic Surgery, Department of Surgery, The Warren Alpert School of Medicine and Rhode Island Hospital, Providence, Rhode Island, USA

Correction notice The article has been corrected since it was published online. The affiliations of the authors were incorrect which have been updated.

Acknowledgements We would like to acknowledge all WHI investigators and participants.

Contributors $\mathrm{MH}$ analysed the data, drafted and revised the manuscript. $\mathrm{KL}$ and $\mathrm{JL}$ assisted in data analysis and manuscript revision. MA, WCW and SL critically reviewed the analyses and revised the manuscript. SL obtained funding for the work, oversaw the entire process of study design, data analysis, manuscript drafting and revisions. All authors read, edited and approved the final manuscript, and have taken due care to ensure its integrity. MH, KL, JL, MA, WCW and SL participated in the study design; searched the literature; collected, analysed and interpreted the data; and wrote the manuscript.

Funding MH and SL were partly supported by fund donated to Brown University by Barilla. The Women's Health Initiative (WHI) programme was funded by the National Heart, Lung and Blood Institute (NHLBI), the National Institutes of Health and the US Department of Health and Human Services through contracts HHSN268201100046C, HHSN268201100001C, HHSN268201100002C, HSN268201100003C, HHSN268201100004C and HHSN271201100004C.

\section{R01DK125403}

Disclaimer The funding sources had no role in study design, data collection, data analysis, data interpretation or the writing of this report, and in the decision to submit the manuscript for publication.

Competing interests None declared.

Patient consent for publication Not required.

Ethics approval Ethical approval of all protocols was obtained from the institutional review boards (IRBs) of all participating institutions (40 clinical site IRBs, the coordinating centre IRB and ethical review at the National Institutes of Health). Please refer to the following website for more information: https://www. whi.org/about/SitePages/StudyOrganization.aspx.

Provenance and peer review Not commissioned; externally peer reviewed.
Data availability statement Data will make avaviable subject to distrubtion policiy of the Women's Health Initiative (WHI) research program and the National Institute of Health (NIH).

Supplemental material This content has been supplied by the author(s). It has not been vetted by BMJ Publishing Group Limited (BMJ) and may not have been peer-reviewed. Any opinions or recommendations discussed are solely those of the author(s) and are not endorsed by BMJ. BMJ disclaims all liability and responsibility arising from any reliance placed on the content. Where the content includes any translated material, BMJ does not warrant the accuracy and reliability of the translations (including but not limited to local regulations, clinical guidelines, terminology, drug names and drug dosages), and is not responsible for any error and/or omissions arising from translation and adaptation or otherwise.

Open access This is an open access article distributed in accordance with the Creative Commons Attribution Non Commercial (CC BY-NC 4.0) license, which permits others to distribute, remix, adapt, build upon this work non-commercially, and license their derivative works on different terms, provided the original work is properly cited, appropriate credit is given, any changes made indicated, and the use is non-commercial. See: http://creativecommons.org/licenses/by-nc/4.0/.

\section{ORCID iDs}

Kenneth Lo http://orcid.org/0000-0003-4624-2737

Jie Li http://orcid.org/0000-0002-7529-6040

Simin Liu http://orcid.org/0000-0003-2098-3844

\section{REFERENCES}

1 Jenkins DJ, Wolever TM, Jenkins AL, et al. Glycemic response to wheat products: reduced response to pasta but no effect of fiber. Diabetes Care 1983;6:155-9.

2 Rasmussen O, Winther E, Arnfred J, et al. Comparison of blood glucose and insulin responses in non-insulin dependent diabetic patients. Studies with spaghetti and potato taken alone and as part of a mixed meal. Eur J Clin Nutr 1988;42:953-61.

3 Parillo M, Giacco R, Riccardi G, et al. Different glycaemic responses to pasta, bread, and potatoes in diabetic patients. Diabet Med 1985;2:374-7.

4 Riccardi G, Clemente G, Giacco R. Glycemic index of local foods and diets: the Mediterranean experience. Nutr Rev 2003;61:S56-60.

5 Hermansen K, Rasmussen O, Arnfred J, et al. Differential glycaemic effects of potato, rice and spaghetti in type 1 (insulindependent) diabetic patients at constant insulinaemia. Diabetologia 1986;29:358-61.

6 Juntunen KS, Niskanen LK, Liukkonen KH, et al. Postprandial glucose, insulin, and incretin responses to grain products in healthy subjects. Am J Clin Nutr 2002;75:254-62.

7 Wolever TM, Jenkins DJ, Kalmusky J, et al. Glycemic response to pasta: effect of surface area, degree of cooking, and protein enrichment. Diabetes Care 1986;9:401-4.

8 Granfeldt Y, Björck I, Hagander B. On the importance of processing conditions, product thickness and egg addition for the glycaemic and hormonal responses to pasta: a comparison with bread made from 'pasta ingredients'. Eur J Clin Nutr 1991;45:489-99.

9 Augustin LSA, Kendall CWC, Jenkins DJA, et al. Glycemic index, glycemic load and glycemic response: an international scientific consensus Summit from the International carbohydrate quality Consortium (ICQC). Nutr Metab Cardiovasc Dis 2015;25:795-815.

10 Juanola-Falgarona M, Salas-Salvadó J, Buil-Cosiales P, et al. Dietary glycemic index and glycemic load are positively associated with risk of developing metabolic syndrome in middle-aged and elderly adults. J Am Geriatr Soc 2015;63:1991-2000.

11 Salmerón J, Manson JE, Stampfer MJ, et al. Dietary fiber, glycemic load, and risk of non-insulin-dependent diabetes mellitus in women. JAMA 1997;277:472-7.

12 Schulze MB, Liu S, Rimm EB, et al. Glycemic index, glycemic load, and dietary fiber intake and incidence of type 2 diabetes in younger and middle-aged women. Am J Clin Nutr 2004;80:348-56.

13 Bhupathiraju SN, Tobias DK, Malik VS, et al. Glycemic index, glycemic load, and risk of type 2 diabetes: results from 3 large US cohorts and an updated meta-analysis. Am J Clin Nutr 2014;100:218-32.

14 Liu S, Willett WC, Stampfer MJ, et al. A prospective study of dietary glycemic load, carbohydrate intake, and risk of coronary heart disease in US women. Am J Clin Nutr 2000;71:1455-61.

15 Liu S, Manson JE, Stampfer MJ, et al. Dietary glycemic load assessed by food-frequency questionnaire in relation to plasma high- 
density-lipoprotein cholesterol and fasting plasma triacylglycerols in postmenopausal women. Am J Clin Nutr 2001;73:560-6.

16 Shikany JM, Tinker LF, Neuhouser ML, et al. Association of glycemic load with cardiovascular disease risk factors: the women's health Initiative observational study. Nutrition 2010;26:641-7.

17 Ford ES, Liu S. Glycemic index and serum high-density lipoprotein cholesterol concentration among US adults. Arch Intern Med 2001;161:572-6.

18 Liu S, Manson JE, Buring JE, et al. Relation between a diet with a high glycemic load and plasma concentrations of high-sensitivity C-reactive protein in middle-aged women. Am J Clin Nutr 2002;75:492-8.

19 Riccardi G, Rivellese AA, Giacco R. Role of glycemic index and glycemic load in the healthy state, in prediabetes, and in diabetes. Am J Clin Nutr 2008;87:269S-74.

20 Patterson RE, Kristal AR, Tinker LF, et al. Measurement characteristics of the women's health Initiative food frequency questionnaire. Ann Epidemiol 1999;9:178-87.

21 Liu S, Tinker L, Song Y, et al. A prospective study of inflammatory cytokines and diabetes mellitus in a multiethnic cohort of postmenopausal women. Arch Intern Med 2007;167:1676-85.

22 Margolis KL, Qi L, Brzyski R. Validity of diabetes self-reports in the women's health Initiative: comparison with medication inventories and fasting glucose measurements. Clin Trials 2008;5:240-7.

23 Goff DC, Lloyd-Jones DM, Bennett G, et al. 2013 ACC/AHA guideline on the assessment of cardiovascular risk: a report of the American College of Cardiology/American heart association Task force on practice guidelines. J Am Coll Cardiol 2014;63:2935-59.

24 Prentice RL, Thomson CA, Caan B, et al. Low-Fat dietary pattern and cancer incidence in the women's health Initiative dietary modification randomized controlled trial. J Natl Cancer Inst 2007:99:1534-43.

25 Brown CC, Kipnis V, Freedman LS, et al. Energy adjustment methods for nutritional epidemiology: the effect of categorization. Am J Epidemiol 1994;139:323-38

26 Grambsch PM, Therneau TM. Proportional hazards tests and diagnostics based on weighted residuals. Biometrika 1994;81:515-26.

27 Guenther PM, Reedy J, Krebs-Smith SM. Development of the healthy eating Index-2005. J Am Diet Assoc 2008;108:1896-901.

28 Huang M, Quddus A, Stinson L, et al. Artificially sweetened beverages, sugar-sweetened beverages, plain water, and incident diabetes mellitus in postmenopausal women: the prospective women's health Initiative observational study. Am J Clin Nutr 2017;106:614-22.

29 R Core Team. R: a language and environment for statistical computing. Vienna, Austria: R Foundation for Statistical Computing, 2020. https://www.R-project.org/

30 Pounis G, Castelnuovo AD, Costanzo S, et al. Association of pasta consumption with body mass index and waist-to-hip ratio: results from Moli-sani and INHES studies. Nutr Diabetes 2016;6:e218.

31 Martínez-González MA, Salas-Salvadó J, Estruch R, et al. Benefits of the Mediterranean diet: insights from the PREDIMED study. Prog Cardiovasc Dis 2015;58:50-60.
32 Estruch R, Ros E, Salas-Salvadó J, et al. Primary prevention of cardiovascular disease with a Mediterranean diet supplemented with extra-virgin olive oil or nuts. N Engl J Med 2018;378:e34.

33 Shay CM, Van Horn L, Stamler J, et al. Food and nutrient intakes and their associations with lower BMI in middle-aged us adults: the International study of Macro-/Micronutrients and blood pressure (INTERMAP). Am J Clin Nutr 2012;96:483-91.

34 Pala V, Sieri S, Masala G, et al. Associations between dietary pattern and lifestyle, anthropometry and other health indicators in the elderly participants of the EPIC-Italy cohort. Nutr Metab Cardiovasc Dis 2006;16:186-201.

35 Mertens E, Markey O, Geleijnse J, et al. Dietary patterns in relation to cardiovascular disease incidence and risk markers in a middle-aged British male population: data from the Caerphilly prospective study. Nutrients 2017;9:75

36 Nettleton JA, Polak JF, Tracy R, et al. Dietary patterns and incident cardiovascular disease in the multi-ethnic study of atherosclerosis. Am J Clin Nutr 2009:90:647-54.

37 Salmerón J, Ascherio A, Rimm EB, et al. Dietary fiber, glycemic load, and risk of NIDDM in men. Diabetes Care 1997;20:545-50.

38 Livesey G, Taylor R, Livesey $\mathrm{H}$, et al. Is there a dose-response relation of dietary glycemic load to risk of type 2 diabetes? meta-analysis of prospective cohort studies. Am J Clin Nutr 2013;97:584-96.

39 Mirrahimi A, de Souza RJ, Chiavaroli L, et al. Associations of glycemic index and load with coronary heart disease events: a systematic review and meta-analysis of prospective cohorts. J Am Heart Assoc 2012;1:e000752.

40 Yu D, Shu X-O, Li H, et al. Dietary carbohydrates, refined grains, glycemic load, and risk of coronary heart disease in Chinese adults. Am J Epidemiol 2013;178:1542-9.

41 Hardy DS, Hoelscher DM, Aragaki C, et al. Association of glycemic index and glycemic load with risk of incident coronary heart disease among whites and African Americans with and without type 2 diabetes: the Atherosclerosis risk in Communities study. Ann Epidemiol 2010;20:610-6.

42 Fan J, Song Y, Wang Y, et al. Dietary glycemic index, glycemic load, and risk of coronary heart disease, stroke, and stroke mortality: a systematic review with meta-analysis. PLoS One 2012;7:e52182.

43 Sieri S, Brighenti F, Agnoli C, et al. Dietary glycemic load and glycemic index and risk of cerebrovascular disease in the EPICOR cohort. PLoS One 2013;8:e62625.

44 Chiavaroli L, Kendall CWC, Braunstein CR, et al. Effect of pasta in the context of low-glycaemic index dietary patterns on body weight and markers of adiposity: a systematic review and metaanalysis of randomised controlled trials in adults. BMJ Open 2018;8:e019438.

45 Vitale M, Masulli M, Rivellese AA, et al. Pasta consumption and connected dietary habits: associations with glucose control, adiposity measures, and cardiovascular risk factors in people with type 2 Diabetes-TOSCA.IT study. Nutrients 2019;12:101. 